Vortex pairs in nonlocal nonlinear media

This article has been downloaded from IOPscience. Please scroll down to see the full text article.

2012 J. Opt. 14065204

(http://iopscience.iop.org/2040-8986/14/6/065204)

View the table of contents for this issue, or go to the journal homepage for more

Download details:

IP Address: 58.198.96.70

The article was downloaded on 19/05/2012 at 06:23

Please note that terms and conditions apply. 


\title{
Vortex pairs in nonlocal nonlinear media
}

\author{
Ming Shen ${ }^{1,2,3}$, YuanYao Lin ${ }^{1}$, Chien-Chung Jeng ${ }^{2}$ and Ray-Kuang Lee ${ }^{1}$ \\ ${ }^{1}$ Institute of Photonics Technologies, National Tsing-Hua University, Hsinchu 300, Taiwan \\ 2 Department of Physics, National Chung-Hsing University, Taichung 402, Taiwan \\ ${ }^{3}$ Department of Physics, Shanghai University, Shanghai 200444, People's Republic of China \\ E-mail: shenmingluck@shu.edu.cn
}

Received 9 January 2012, accepted for publication 25 April 2012

Published 18 May 2012

Online at stacks.iop.org/JOpt/14/065204

\begin{abstract}
Dynamics of vortex pairs with the same and opposite circulations are studied theoretically in nonlocal nonlinear media with different intervortex separations. We demonstrate that the nonlocal nonlinear response not only leads to a dramatic suppression of the elliptical instabilities but also helps in the formation of quasi-stable rotating and breathing bound states for vortex-vortex and vortex-antivortex (vortex dipole) pairs, respectively.
\end{abstract}

Keywords: nonlocal nonlinear media, vortex dipoles, azimuthal instability

(Some figures may appear in colour only in the online journal)

\section{Introduction}

During the past decade, nonlocal solitons have been a hot subject because the nonlocality has a profound impact on the stabilization of soliton formations [1]. Nonlocality is an inherent property in many different fields of physics. Nonlocal nonlinearity can be found in various systems, such as lead glass with thermal conduction [2, 3], atomic vapors with diffusion of charge carriers [4] and photorefractive materials with the drift of photoexcited charges [5]. Nonlocality can also be represented as a long-range interaction of constituent molecules or particles. This happens, for example, in nematic liquid crystals with a reorientational nonlinearity [6, 7] or in Bose-Einstein condensates (BECs) with different types of long-range interactions [8-10].

In optics, many works have shown that nonlocality can drastically affect the propagation of spatially optical solitons. Nonlocality can support stable dipole [11, 12] and multi-pole solitons [13, 14]. In optical lattices with a nonlocal nonlinearity, some unique properties of the propagation of solitons, such as self-bending [15], mobility [16] and gap solitons [17-19], have been investigated extensively. The nonlocality can provide a long-range force between solitons [3], leading to the formation of bound states for both out-of-phase bright [20, 21] and dark solitons [22-24]. Experimental and theoretical studies have shown that the nonlocality plays an important role for incoherent solitons [25-29]. The nonlocality can suppress the modulation instability [30, 31] and transverse instability [32], and prevent the catastrophic collapse of high-dimensional optical beams [33]. Nonlocal nonlinearity also sustains vector coupled solitons, including vector dipole soliton pairs [34, 35], multi-pole vector solitons [36, 37], two-color vector solitons [38-40], bright and dark solitons [41], vector vortex [42] and necklace solitons [43].

It is known that a vortex is a universal signature of phase singularity, which has been studied in different areas of both linear [44] and nonlinear systems [45] from classical fluids to ultracold quantum gases. In optics, propagation and manipulation of vortices have been investigated extensively in various nonlinear media for their possible applications in optical communication and quantum information processing [46]. In local media, the light-induced refractive index change is a local function of the light intensity. Because of azimuthal instability, the vortex solitons will split and break up [47], which can only be stable in media with the introduction of competing nonlinearities [48]. However, in nonlocal media, the nonlinear response of the medium in a particular location depends on the light intensity in a certain neighborhood of this location [1]. Due to the long-range interactions, the nonlocality can surely support such stable vortex structures with different angular momenta, such as azimuthons [49-51] and vortex solitons [2, 52-54]. The stabilization and dynamics of vortex solitons are solely determined by the nonlocal response function [55-59].

Although a single vortex carries orbital angular momentum, vortex pairs, vortex-vortex or vortex-antivortex (vortex 
dipole), undergo different instabilities beyond a coherent superposition. A pair of vortices with the same circulations is energetically unfavorable when considered as a higher-order vortex. Only vortex pairs with opposite circulations can be stable, which forms a bound state [60]. Instead, vortex dipoles, a pair of vortices with equal and opposite circulations, are a basic topological structure to carry linear momentum. Recently, vortex dipoles have drawn considerable attention in several different physical systems, including optics, BECs and exciton-polariton condensates [61]. In optics, the dipole soliton vortex can be considered as coherent states of dipole solitons carrying a nonzero topological charge [62]. The experimental observation of a fractional vortex dipole has been demonstrated in 2009 [63]. In very recent years, the vortex dipoles are also demonstrated in BECs [64, 65], with anisotropic dipolar-dipolar nonlocal interactions [66].

It is also known that a nonzero intervortex separation in a vortex pair would induce a symmetry breaking in the translational direction, i.e. the elliptical instability [67, 68] which can scale the strain imposed on each vortex core. As a vortex pair is formed with a nonzero separation, it was revealed in the fluid dynamics that the streamlines in the core of each vortex have an elliptical shape for the involved Kelvin modes, for which the related instabilities are coined as elliptical instability [67]. To provide a deeper understanding of superfluid phenomena, in this paper, we demonstrate that the nonlocal nonlinear response leads to a dramatic suppression of the elliptical instabilities both for pairs of vortices with the same and opposite circulations. Moreover, with a close analogy to point vortices in a fluid, we reveals that vortex-vortex and vortex-antivortex pairs in strong nonlocal nonlinear media evolve into quasi-stable rotating and breathing bound states, respectively.

\section{Basic model and equation}

In nonlocal nonlinear media the propagation of a paraxial optical beam with the scalar field envelope $\psi$ is governed by the nonlinear Schrödinger equation

$$
\mathrm{i} \frac{\partial \psi}{\partial z}+\frac{\partial^{2} \psi}{\partial x^{2}}+\frac{\partial^{2} \psi}{\partial y^{2}}+\psi \int R\left(\mathbf{r}-\mathbf{r}^{\prime}\right) I\left(\mathbf{r}^{\prime}, z\right) \mathrm{d}^{2} \mathbf{r}^{\prime}=0
$$

where $I(\mathbf{r}, z)=|\psi(x, z)|^{2}$ is the beam intensity and $R(\mathbf{r})$ is the nonlocal response function with the degree of nonlocality denoted by $\sigma$ [30]. Generally, the nonlocal response is determined by the physics of the underlying nonlinear process $[2,4,6,8]$.

We first seek analytically the approximate solution of the vortex pairs by using the variational approach through the Lagrangian density of equation (1), i.e.

$$
\begin{aligned}
\mathcal{L}= & \frac{\mathrm{i}}{2}\left(\psi^{*} \frac{\partial \psi}{\partial z}-\psi \frac{\partial \psi^{*}}{\partial z}\right)-\left(\left|\frac{\partial \psi}{\partial x}\right|^{2}+\left|\frac{\partial \psi}{\partial y}\right|^{2}\right) \\
& +\frac{1}{2}|\psi|^{2} \int R\left(\mathbf{r}-\mathbf{r}^{\prime}\right) I\left(\mathbf{r}^{\prime}, z\right) \mathrm{d}^{2} \mathbf{r}^{\prime} .
\end{aligned}
$$

The most important issue of the variational approach is to choose an appropriate ansatz of the optical vortex dipole solitons. Compared with an optical vortex, the similar phenomena of vortex dipoles, tripoles, quadrupoles and clusters have also been investigated previously in Bose-Einstein condensation $[69,70]$. Thus, in this work, we take the following class of variational ansatz for the optical vortex dipole solitons, which was used to study the vortex and antivortex pairs in a trapped two-dimensional BEC [71]:

$$
\psi=A(x-a+\mathrm{i} y)(x+a \pm \mathrm{i} y) \operatorname{Exp}\left(-\frac{x^{2}+y^{2}}{2 w^{2}}+\mathrm{i} k z\right),
$$

where $A, w$ and $k$ are the amplitude, beam width and wavenumber of the vortex, respectively. A vortex-vortex (vortex-antivortex) pair is denoted by the $+(-)$ sign, corresponding to a pair of vortices with equal and the same (opposite) circulation, and $a$ is related to the separation of two vortex cores. For convenience, we also consider here the case of a Gaussian nonlocal response [54]:

$$
R(\mathbf{r})=\left(\pi \sigma^{2}\right)^{-1} \exp \left(-\mathbf{r}^{2} / \sigma^{2}\right) .
$$

Although the Gaussian response nonlocality is not a realistic known physical system, it can describe the behaviors of solitons in nonlocal media physically and simply. It has also been shown that, as long as the response function is monotonically decaying, the physical properties of soliton solutions do not depend strongly on its shape [72].

The analytical results are summarized in figure 1 , where we plot the powers for vortex-vortex and vortex-antivortex pairs versus the beam width $w$ and wavenumber $k$, respectively. From figure 1(a), one can see that, when two vortices are overlapping exactly, $a=0$, the formation power decreases as the beam width increases, which is similar to the case of a scalar vortex in local and nonlocal media [35]. But with a nonzero intervortex separation, as shown for $a=0.6$, the constructive interference between two vortices makes the power-beam width relation for vortex-vortex pair reach a minimum value around $w=0.5$, which corresponds to the minimum of the Hamiltonian of the system [73]. To avoid a huge formation power of a zero intervortex separation for $a=$ 0 , we fixed the beam width to $w=1$, in order to demonstrate a clear comparison of dynamics for different intervortex separations As shown in figure 1(b), both the powers for vortex-vortex and vortex-antivortex increase when $k$ and $\sigma$ increase.

\section{Vortex pairs with same circulations}

With the variational results, we employ the found ansatz solution as an input for numerical simulations of equation (1) directly by using the split step beam propagation method. In all of our simulations, we set the initial beam width as $w=1$. In figures 2-4, we demonstrate the dynamics and stability properties of vortex-vortex pairs in nonlocal media with various degrees of the nonlocality, i.e. $\sigma=0.1,1.5$ and 10 , respectively. It is known that a weak nonlocality cannot suppress the azimuthal instability of vortex solitons [35, 52]. In this case, as shown in figure 2, vortex-vortex pairs experience a symmetry-breaking instability, split into several 

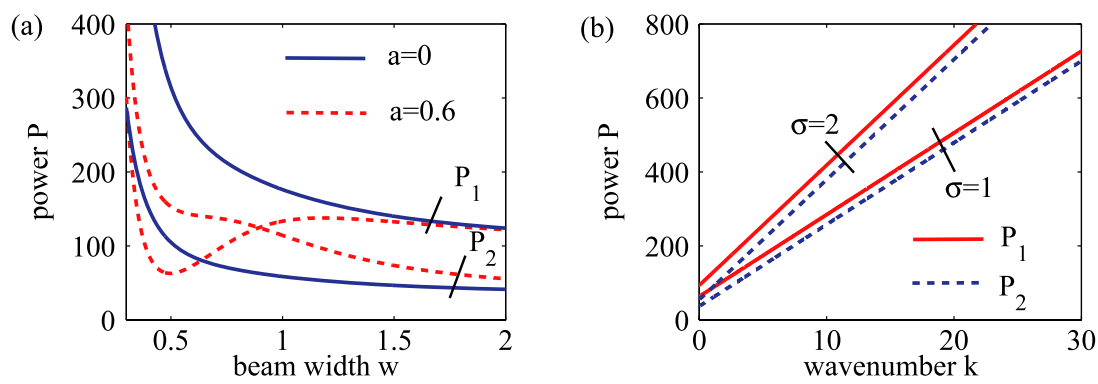

Figure 1. The power of the vortex dipoles versus the beam width (a) and wavenumber (b) with the parameters $\sigma=1$ for (a) and $a=0.6$, $w=1$ for (b). $P_{1}$ and $P_{2}$ are the power of the vortex-vortex and vortex-antivortex pairs, respectively.

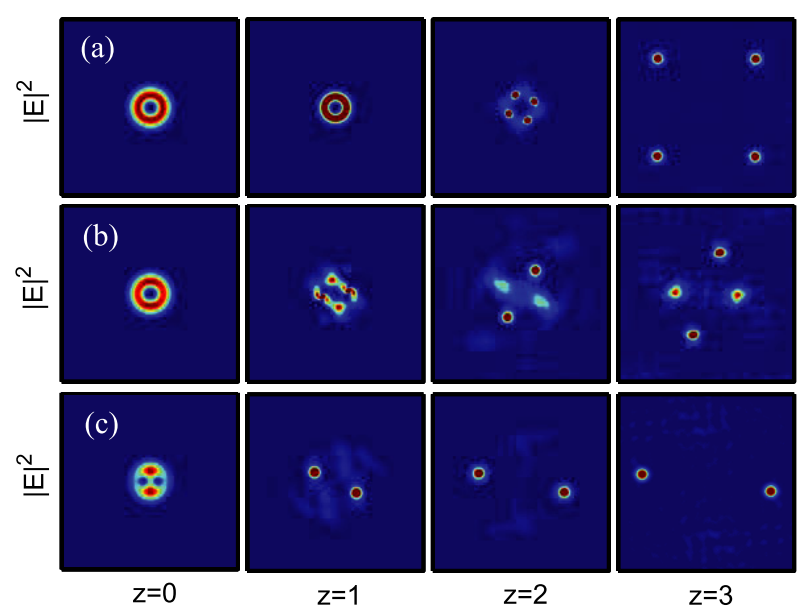

Figure 2. Symmetric breaking of in-phase vortex dipole solitons in weakly nonlocal media with $\sigma=0.1$. The parameters are $a=0$ (a), $a=0.2(\mathrm{~b})$ and $a=0.8(\mathrm{c}) .|E|^{2}$ represents the intensity of vortex dipoles.

filaments, and fly away to each other. However, the breaking dynamics is very different and depends crucially on the intervortex separation parameter $a$. When $a=0$, the intensity of the vortex-vortex pair is uniform along the azimuthal direction, which represents a circularly symmetric vortex with double charges, resulting in four fundamental solitons with equal intensities (figure 2(a)). For a nonzero separation, as shown in figure 2(b) with $a=0.2$, the uniformity of intensity along azimuthal directions is broken: the vortex pair splits into two groups of fundamental solitons with different intensities, When the intervortex separation is comparable to the beam width, for example, $a=0.8$ in figure 2(c), the intensity of the vortex-vortex pair has two peaks in the opposite position, and only splits into two fundamental dipole-like solitons as a manifestation of the elliptic instability of vortex cores [67].

Dynamics of the vortex-vortex pair changes with a moderate degree of nonlocality, which induces a mutual self-trapping to stabilize vortex pairs, as shown in figure 3 for $\sigma=1.5$. When the intervortex separation $a$ is zero and small, it is obvious that the nonlocality can effectively suppress the azimuthal instability of vortex-vortex pairs. For instance, the vortex-vortex pair with $a=0$ is stable at the propagation distance $z=20$ (figure 3(a)) whereas it has already broken at a shorter propagation distance $z=2$ in weakly nonlocal media (figure 2(a)). An interesting dynamics is found with a
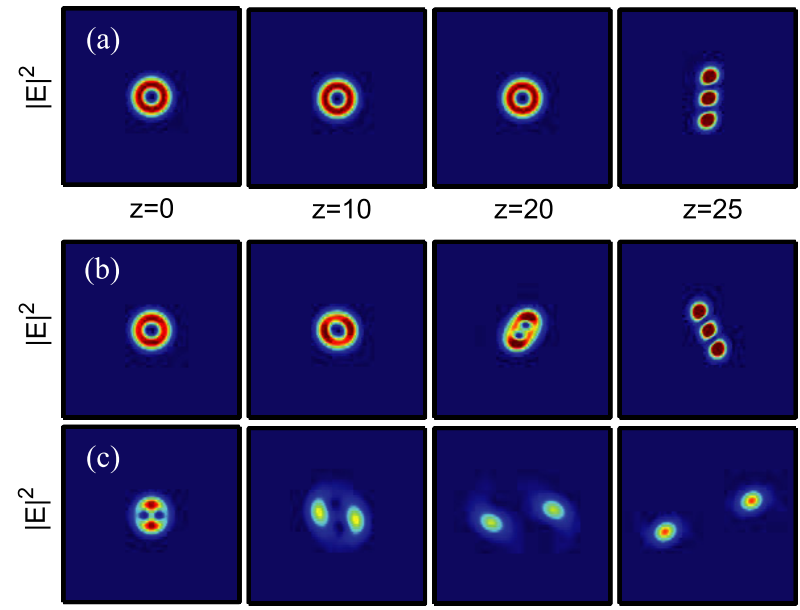

$z=1$

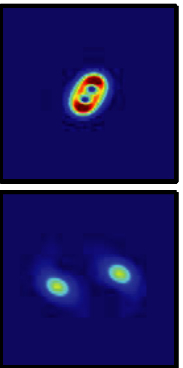

$z=2$

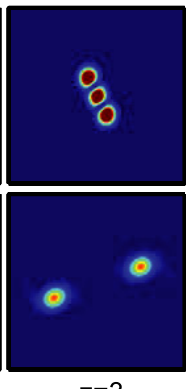

$z=3$
Figure 3. Dynamics of in-phase vortex dipole solitons in moderately nonlocal media with $\sigma=1.5$. The parameters are $a=0$ (a), $a=0.2$ (b) and $a=0.8$ (c).

moderate nonlocality, that a vortex-vortex pair with zero or a small separation only breaks up into three filaments, instead of four filaments in weakly nonlocal media. This result indicates that the nonlocality can suppress the azimuthal instability, resulting in the decrease of the numbers of filament beams. But for a larger value of separation, $a=0.8$ in figure 3(c), we can see that a moderate nonlocality still cannot suppress the splitting and expansion of the vortex-vortex pair because now the induced symmetry breaking comes predominantly from elliptical instability instead of azimuthal instability. This vortex-vortex pair will ensure stability only in the strongly nonlocal regime, as shown in figure 4(c).

We show in figure 4 the quasi-stable propagation of vortex-vortex pairs in strongly nonlocal media with $\sigma=10$. A stronger nonlocality can average out all spatial variations of the intensity distribution and induce an effective attractive potential to suppress the azimuthal instability [43], resulting in the formation of a stationary vortex-vortex pair. When $a=0$ or small $(a=0.2)$, the vortex-vortex pair evolves into a rotating one and 'breathes' along the propagation distance, with a slight modulation of the intensity along the vortex rings, as shown in figures 4(a) and (b). Instead, with a larger separation as shown in figure 4(c) for $a=0.8$, a vortex-vortex pair carries two angular momenta resulting in a nonuniform 


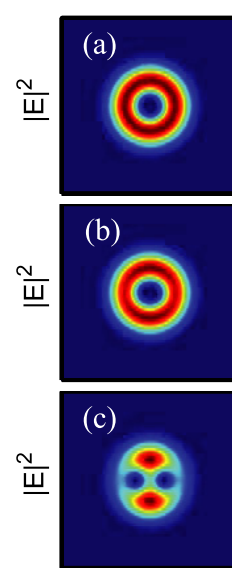

$z=0$

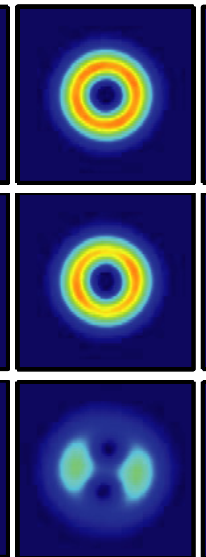

$z=1$

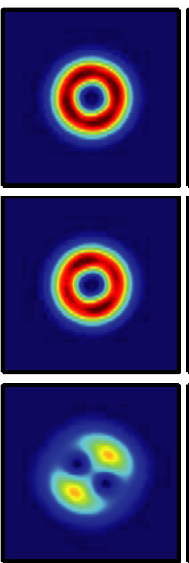

$z=2$

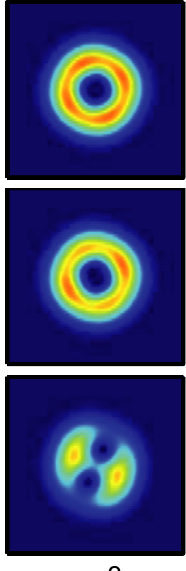

$z=3$
Figure 4. Quasi-stable in-phase vortex dipole solitons in strongly nonlocal media with $\sigma=10$. The parameters are $a=0$ (a), $a=0.2(\mathrm{~b})$ and $a=0.8(\mathrm{c})$.

intensity distribution. Then a quasi-stable bound state for the vortex-vortex pair with a dipolar intensity distribution can survive, which possesses a counterclockwise rotation along the propagation distance. It should be emphasized that previous work has demonstrated the similar phenomena of rotation also happened to multi-vortex solitons in nonlocal media [59].

\section{Vortex pairs with opposite circulations: vortex dipoles}

By reversing the sign in equation (3), the nonlinear dynamics for vortex-antivortex pairs, vortex dipoles, are totally different. In figure 5, we demonstrate the comparison of dynamics for vortex-antivortex pairs in weakly (figures 5(a) and (c) with $\sigma=0.1$ ) and strongly nonlocal media (figures 5(b) and (d) with $\sigma=10$ ), respectively. Unlike the counterpart of vortex-vortex pairs, with a zero separation $a=$ 0 , a vortex-antivortex pair does not break up into filaments but decays into a quasi-stable bright soliton (figure 5(a)). According to equation (3) the total field distribution for a vortex-antivortex pair represents a beam without carrying any angular momentum. A similar scenario happens for vortex-antivortex pairs with a stronger degree of nonlocality (figure 5(b)). Thus a vortex-antivortex pair will not split by the azimuthal instability but only decay into a quasi-stable bright soliton.

In contrast, the cancellation of angular momentum for a vortex-antivortex pair with a larger separation turns its propagation into two filaments in a weakly nonlocal medium (figure 5(c)), as a vortex-vortex pair shown in (figure 2(c)), but moving towards the $-y$ direction. We also numerically confirmed that the moving direction is always perpendicular to the connection direction of the two vortex core when two vortices with contrary angular momentum were placed along an arbitrary direction (not shown). The motion of such a vortex-antivortex pair comes from the nonvanishing effective linear momentum, i.e. for the separation set in the $x$ direction as shown in equation (3). As for a positive or negative $y$

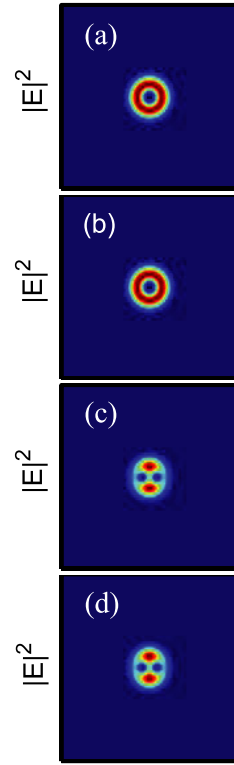

$\mathrm{z}=0$

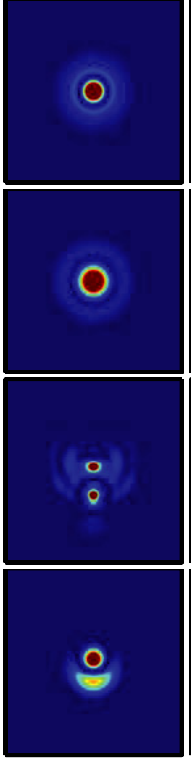

$z=1$

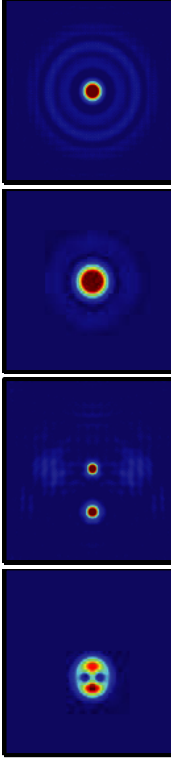

$z=2$

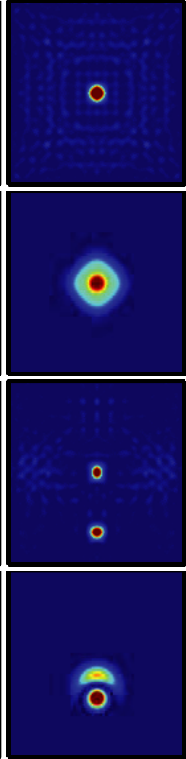

$z=3$
Figure 5. Dynamics of out-of-phase vortex dipoles in weakly $(\sigma=0.1$ for (a) and (c)) and strongly nonlocal media $(\sigma=10$ for (b) and (d)). The parameters are $a=0$ (a) and (b), and $a=0.8$ (c) and (d).

direction, there should be no preference, but in numerical simulations, such a symmetry-breaking phenomenon comes from our numerical method used. In the strongly nonlocal limit, instead of forming a rotating bound state shown in figure 4(c), a quasi-stable breathing bound state is revealed for vortex-antivortex pairs as shown in figure $5(\mathrm{~d})$ with $a=0.8$. The nonlocality of the nonlinear medium response supports quasi-periodic transformations between different symmetries of self-trapped optical beams [74]. By considering the nonlinear system approximately into a linear one in the strongly nonlocal limit, such a breathing bound state, with a periodically changing but asymmetric profile, can be viewed as superpositions of Laguerre-Gaussian beams.

Before the conclusion, we would like to address the comparison of our current work to previous studies on the dynamics of vortex solitons in nonlocal media. In [58], the authors studied vortices in strong nonlocal nonlinear media (thermal optical nonlinearity) which are subject to the preset boundary. Buccoliero et al considered a general vortex in the form of generalized Gaussian beams in nonlocal media and showed the spiraling of a particular array of vortices under propagation [59]. In contrast, we emphasize that, in this paper, our current work aims at the dynamics of vortex-vortex and vortex-antivortex pairs, which differs considerably from these earlier studies. On the one hand, the link for this system to elliptical instability of vortex-antivortex pairs is first revealed here and, on the other hand, this work also reveals the formation of moving (in the transverse direction) the quasi-stable bright soliton from the vortex-antivortex pair under the balance between linear momentum and strong nonlocality. 


\section{Conclusion}

In summary, we demonstrate a variety of dynamics both for vortex-vortex and vortex-antivortex pairs in nonlocal nonlinear media. In addition to the suppression of azimuthal instability, we show that the nonlocal nonlinear response also suppress elliptical instability and forms quasi-stable rotating or breathing states for vortex pairs depending on their circulations.

\section{Acknowledgments}

This work is supported in part by the Chinese NSFC (60808002) and the Shanghai Leading Academic Discipline Project (S30105).

\section{References}

[1] Krolikowski W, Bang O, Nikolov N I, Neshev D, Wyller J, Rasmussen J J and Edmundson D 2004 J. Opt. B 6 S288

[2] Rotschild C, Cohen O, Manela O, Segev M and Carmon T 2005 Phys. Rev. Lett. 95213904

[3] Rotschild C, Alfassi B, Cohen O and Segev M 2006 Nature Phys. 2769

[4] Skupin S, Saffman M and Krolikowski W 2007 Phys. Rev. Lett. 98263902

[5] Cohen O, Bartal G, Buljan H, Carmon T, Fleischer J W, Segev M and Christodoulides D N 2005 Nature 433500

[6] Peccianti M, Conti C, Assanto G, Luca A D and Umeton C 2004 Nature 432733

[7] Peccianti M, Dyadyusha A, Kaczmarek M and Assanto G 2006 Nature Phys. 2737

[8] Pedri P and Santos L 2005 Phys. Rev. Lett. 95200404

[9] Maucher F, Skupin S, Shen M and Krolikowski W 2010 Phys. Rev. A 81063617

[10] Maucher F, Henkel N, Saffman M, Krolikowski W, Skupin S and Pohl T 2011 Phys. Rev. Lett. 106170401

[11] Lopez-Aguayo S, Desyatnikov A S, Kivshar Yu S, Skupin S, Krolikowski W and Bang O 2006 Opt. Lett. 311100

[12] Ye F, Kartashov Y V and Torner L 2008 Phys. Rev. A 77043821

[13] Rotschild C, Segev M, Xu Z, Kartashov Y V, Torner L and Cohen O 2006 Opt. Lett. 313312

[14] Xu Z, Kartashov Y and Torner L 2005 Opt. Lett. 303171

[15] Kartashov Y V, Vysloukh V A and Torner L 2004 Phys. Rev. Lett. 93153903

[16] Xu Z, Kartashov Y V and Torner L 2005 Phys. Rev. Lett. 95113901

[17] Lin Y Y, Lee R-K and Malomed B A 2009 Phys. Rev. A 80013838

[18] Lin Y Y, Jisha C P, Jeng C J, Lee R-K and Malomed B A 2010 Phys. Rev. A 81063803

[19] Kuo K-H, Lin Y Y, Lee R-K and Malomed B A 2011 Phys. Rev. A 83053838

[20] Peccianti M, Brzdakiewicz K and Assanto G 2002 Opt. Lett. 271460

[21] Rasmussen P D, Bang O and Krolikowski W 2005 Phys. Rev. E 72066611

[22] Nikolov N, Neshev D, Krolikowski W, Bang O, Rasmussen J and Christiansen P 2004 Opt. Lett. 29286

[23] Dreischuh A, Neshev D N, Petersen D E, Bang O and Krolikowski W 2006 Phys. Rev. Lett. 96043901

[24] Kong Q, Wang Q, Bang O and Krolikowski W 2010 Phys. Rev. A 82013826

[25] Krolikowski W, Bang O and Wyller J 2004 Phys. Rev. E 70036617
[26] Cohen O, Buljan H, Schwartz T, Fleischer J W and Segev M 2006 Phys. Rev. E 73 015601(R)

[27] Shen M, Wang Q, Shi J, Chen Y and Wang X 2005 Phys. Rev. E 72026604

[28] Rotschild C, Schwartz T, Cohen O and Segev M 2008 Nature Photon. 2371

[29] Alfassi B, Rotschild C and Segev M 2009 Phys. Rev. A 80 041808(R)

[30] Krolikowski W, Bang O, Rasmussen J J and Wyller J 2001 Phys. Rev. E 64016612

[31] Wyller J, Krolikowski W, Bang O and Rasmussen J J 2002 Phys. Rev. E 66066615

[32] Lin Y Y, Lee R-K and Kivshar Yu S 2008 J. Opt. Soc. Am. B 25576

[33] Bang O, Krolikowski W, Wyller J and Rasmussen J J 2002 Phys. Rev. E 66046619

[34] Shen M, Chen X, Shi J, Wang Q and Krolikowski W 2009 Opt. Commun. 2824805

[35] Shen M, Ding H, Kong Q, Ruan L, Pang P, Shi J and Wang Q 2010 Phys. Rev. A 82043815

[36] Kartashov Y V, Torner L, Vysloukh V A and Mihalache D 2006 Opt. Lett. 311483

[37] Xu Z, Kartashov Y V and Torner L 2006 Phys. Rev. E 73 055601(R)

[38] Alberucci A, Peccianti M, Assanto G, Dyadyusha A and Kaczmarek M 2006 Phys. Rev. Lett. 97153903

[39] Skuse B D and Smyth N F 2008 Phys. Rev. A 77013817

[40] Shen M, Kong Q, Shi J and Wang Q 2008 Phys. Rev. A 77015811

[41] Lin Y and Lee R 2007 Opt. Express 158781

[42] Xu Z, Smyth N F, Minzoni A A and Kivshar Y S 2009 opt. Lett. 341414

[43] Shen M, Kong Q, Jeng C-C, Ge L-J, Lee R-K and Królikowski W 2011 Phys. Rev. A 83023825

[44] Dennis M R, O'Holleran K and Padgett M J 2009 Prog. Opt. $\mathbf{5 3} 293$

[45] Kivshar Y S and Agrawal G P 2003 Optical Solitons: From Fibers to Photonic Crystals (London: Academic)

[46] Yao A M and Padgett M J 2011 Adv. Opt. Photon. 3161

[47] Firth W J and Skryabin D V 1997 Phys. Rev. Lett. 792450

[48] Desyatnikov A S, Kivshar Y S and Torner L 2005 Prog. Opt. 47291

[49] Lopez-Aguayo S, Desyatnikov A S and Kivshar Yu S 2006 Opt. Express 147903

[50] Skupin S, Grech M and Krolikowski W 2008 Opt. Express 169118

[51] Maucher F, Buccoliero D, Skupin S, Grech M, Desyatnikov A and Krolikowski W 2009 Opt. Quantum Electron. 41337

[52] Briedis D, Petersen D, Edmundson D, Krolikowski W and Bang O 2005 Opt. Express 13435

[53] Minzoni A A, Smyth N F, Worthy A L and Kivshar Y S 2007 Phys. Rev. A 76063803

[54] Buccoliero D, Desyatnikov A S, Krolikowski W and Kivshar Yu S 2007 Phys. Rev. Lett. 98053901

[55] Skupin S, Bang O, Edmundson D and Krolikowski W 2006 Phys. Rev. E 73066603

[56] Yakimenko A I, Zaliznyak Y A and Kivshar Y S 2005 Phys. Rev. E 71 065603(R)

[57] Kartashov Y V, Vysloukh V A and Torner L 2007 Opt. Express 159378

[58] Ye F, Kartashov Y V, Hu B and Torner L 2010 Opt. Lett. 35628

[59] Buccoliero D, Desyatnikov A S, Krolikowski W and Kivshar Yu S 2008 Opt. Lett. 33198

[60] Mamaev A V, Saffman M and Zozulya A A 1996 Phys. Rev. Lett. 774544

[61] Roumpos G, Fraser M D, Löffler A, Höfling S, Forchel A and Yamamoto Y 2011 Nature Phys. 7129 
[62] Kartashov Y V, Ferrando A and Garcia-March M-A 2007 Opt. Lett. 322155

[63] Maleshkov G, Neshev D N and Dreischuh A 2009 Phys. Rev. A 80053828

[64] Neely T W, Samson E C, Bradley A S, Davis M J and Anderson B P 2010 Phys. Rev. Lett. 104160401

[65] Freilich D V, Bianchi D M, Kaufman A M, Langin T K and Hall D S 2010 Science 3291182

[66] Ticknor C, Wilson R M and Bohn J L 2011 Phys. Rev. Lett. 106065301

[67] Leweke T and Williamson C H K 1998 J. Fluid Mech. 36085
[68] Lin Y Y and Lee R-K 2008 Opt. Lett. 331377

[69] Crasovan L-C, Molina-Terriza G, Torres J P, Torner L, Perez-Garcia V M and Mihalache D 2002 Phys. Rev. E 66036612

[70] Crasovan L-C, Vekslerchik V, Perez-Garcia V M, Torres J P Mihalache D and Torner L 2003 Phys. Rev. A 68063609

[71] Li W, Haque M and Komineas S 2008 Phys. Rev. A 77053610

[72] Zhong W and Belic M 2009 Phys. Rev. A 79023804

[73] Desyatnikov A S and Kivshar Yu S 2002 Phys. Rev. Lett. 88053901

[74] Buccoliero D and Desyatnikov A S 2009 Opt. Express 179608 